\title{
Subwavelength surface-relief gratings for stellar coronagraphy
}

\author{
Dimitri Mawet, Pierre Riaud, Jean Surdej, and Jacques Baudrand
}

\begin{abstract}
We present a new design of a phase mask coronagraph implemented with subwavelength diffractive optical elements consisting of optimized surface-relief gratings. Phase mask coronagraphy is a recent technique that seeks to accommodate both high dynamic and high angular resolution imaging of faint sources around bright astrophysical objects such as exoplanets orbiting their host stars. The original design we propose is a new, integrated, and flexible solution to the $\pi$ phase-shift chromaticity of the phase mask coronagraphs. It will allow broadband observations, i.e., shorter integration times and object characterizations, by means of spectroscopic analysis. The feasibility of the component manufacturing is also considered through a tolerance study. (C) 2005 Optical Society of America
\end{abstract}

OCIS codes: $220.4830,050.5080$.

\section{Introduction}

Direct detection of faint sources around bright astrophysical objects such as stars or active galactic nuclei is difficult because of the large flux ratio between them. For example, $\beta$-Pictoris debris disk is 1000 times fainter than its host star in the visible spectrum. The study of such objects requires dedicated instruments called coronagraphs. Coronagraphy has been used in high dynamic imaging since its invention by Lyot in $1939 .{ }^{1}$ The Lyot coronagraph principle is simple: it consists of an occulting mask at the telescope focus, which is centered on the bright object, and a well-dimensioned diaphragm, the so-called Lyot stop, in the relayed pupil to remove diffraction residuals. Lyot's design proved to be efficient for solar and stellar coronagraphy ${ }^{2}$ and prolific, giving birth to an entire new family of improved byproducts, the so-called amplitude coronagraphs. Unfortunately, amplitude coronagraphs possess a major inherent weakness: The physical extension of the opaque zone occults quite a significant region centered on the optical axis and thus all the sources behind it. For ex-

D. Mawet (mawet@astro.ulg.ac.be), P. Riaud, and J. Surdej are with the Institut d'Astrophysique et de Géophysique, Université de Liège, 17 Allée du 6 Août, B-4000 Sart Tilman, Belgium. J. Baudrand is with the Observatoire de Paris-Meudon, 5 place Jules Janssen, F-92195 Meudon, France.

Received 28 February 2005; revised manuscript received 23 June 2005; accepted 24 June 2005.

0003-6935/05/347313-09\$15.00/0

(C) 2005 Optical Society of America ample, let us mention the case of the recent notchfilter mask. ${ }^{3}$ This design presents a large central opaque zone extending in the focal plane up to $3 \lambda / D$ ( $D$ is the telescope diameter, whereas $\lambda$ is the wavelength), where the potential companion is still attenuated by at least $50 \%$.

New types of coronagraph have been designed as alternative solutions to the classical amplitude coronagraphs. This family of components acts on the phase instead of acting on the amplitude; they are, therefore, called phase coronagraphs. ${ }^{4}$ The phase coronagraphs are not affected by the inherent central "dead zone" of the amplitude coronagraphs. Rouan et $a l .{ }^{5}$ proposed in 2000 a promising design, the socalled four-quadrant phase mask coronagraph (FQPM). The principle is to divide the focal plane into four equal areas centered on the optical axis, with two of them providing a $\pi$ phase shift. This causes destructive interference ("nulling") to occur inside the geometric pupil area (Fig. 1). The nulling phenomenon's quality determines the coronagraph's performance and is quantified by the value of the so-called "nulling (or null) depth" coefficient over the considered spectral band.

The FQPM principle has been validated on the bench in monochromatic light ${ }^{6}$ and installed on the NAOS-CONICA adaptive optics at the VLT (Very Large Telescope) UT4. It has given promising preliminary scientific results ${ }^{7}$ and perspectives for future instruments such as the James Webb Space Telescope (JWST) (the infrared successor of the Hubble Space Telescope) and VLT-PF (Planet-Finder, a proposal for the second generation instruments of the 


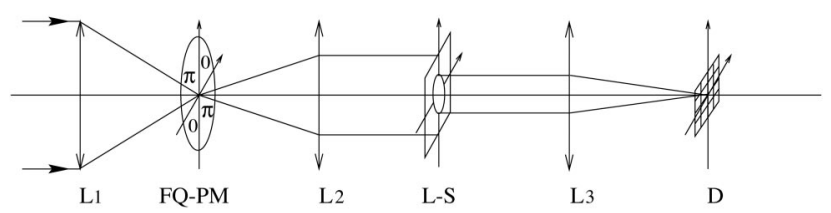

Fig. 1. Basic FQPM coronagraphic optical bench scheme: L1, L2, and L3 are three lenses in the optical system. L1 provides a large (to minimize spatial defects) $F / D$ ratio on the FQPM; L2 images the pupil in the second plane. The Lyot stop (L-S) suppresses the diffracted starlight, and, finally, L3 forms the coronagraphic image on detector $\mathrm{D}$.

Very Large Telescope). Manufacturing challenges of the FQPM come from the $\pi$ phase shift achievement. So far only monochromatic masks have been realized using thin-film deposition or etching techniques on the "index step" principle: a step height $h$ in a material of refractive index $n$ at wavelength $\lambda$ induces a phase shift:

$$
\Delta \phi=\frac{2 \pi}{\lambda}(n-1) h
$$

Unfortunately, this only works perfectly for a given wavelength. The hyperbolic phase-shift dependence in $\lambda$ makes it inapplicable for high-performance use of such devices over a large spectral bandwidth. For instance, the nominal performance of the monochromatic mask used on NAOS-CONICA in the $K$ band $(2-2.4 \mu \mathrm{m})$ under well-corrected atmospheric turbulence is about 2000 times weaker than in monochromatic laboratory experiments. ${ }^{6}$ In this paper we propose an original solution to achromatize and implement the $\pi$ phase shift on the FQPM so it could be used over regular spectral bands corresponding to classical astrophysical filters allowing high nulling performances. Large band observations permit shorter integration times and object characterizations by spectroscopic analysis. Our solution is based on the dispersion of the so-called form birefringence of subwavelength surface-relief gratings.

Section 2 is devoted to the presentation of the zeroorder gratings and to their very specific properties. In Section 3 we will describe the original implementation of the gratings into the final coronagraphic component. The theoretical design will be considered in detail in Section 4, followed by discussion of a tolerance study in Section 5 to demonstrate the manufacturing feasibility. Finally, Section 6 will be dedicated to the coronagraphic performance assessments.

\section{Zero-Order Gratings}

Zero-order gratings (ZOGs) consist of subwavelength gratings, i.e., the period of the structure is smaller than the wavelength of the incident light. They do not diffract light in the sense that only the zero transmitted and reflected orders are allowed to propagate outside the grating region, leaving incident wavefronts free from any further aberrations.

The condition under which a diffraction order prop- agates or not is determined by the well-known grating equation

$$
\frac{\Lambda}{\lambda}=\frac{m}{n_{I} \sin \theta+n_{I, I I I} \sin \theta_{m}},
$$

where $\theta$ is the angle of incidence; $\theta_{m}$ is the exit angle of the $m$ th order of diffraction; and $n_{I}$ and $n_{I I I}$ are the refractive indices of the incident (superstrate) and transmitting (substrate) media, respectively. By convention, the plus sign with the index $n_{I}$ corresponds to the reflection, and the minus sign corresponds to the index $n_{I I I}$ to the transmission. From this relation, we can immediately derive a ZOG condition on the grating period to the wavelength ratio

$$
\frac{\Lambda}{\lambda} \leq \frac{1}{n_{I} \sin \theta+\max \left(n_{I}, n_{I I I}\right)} .
$$

ZOGs present very specific properties: Onedimensionally modulated ZOGs artificially create unique anisotropic and dispersive characteristics that can be used to synthesize achromatic wave plates leading to a vectorial phase difference, i.e., between the vectorial polarization states TE (transverse electric: the electrical field is perpendicular to the plane of incidence, where the plane of incidence is defined by the grating normal and the direction of the incoming light) and TM (transverse magnetic: the electrical field lies in the plane of incidence),

$$
\Delta \phi_{\mathrm{TE}-\mathrm{TM}}=\frac{2 \pi}{\lambda} h \Delta n_{\mathrm{TE}-\mathrm{TM}} .
$$

To simulate grating responses and calculate the socalled form birefringence $\Delta n_{\text {form }}=\Delta n_{\mathrm{TE}-\mathrm{TM}}$ in the subwavelength and resonant domains $(\Lambda \approx \lambda)$, scalar theories of diffraction dramatically fail. The vectorial nature of light must be taken into account. Several approaches are possible depending on the grating period to wavelength ratio. In the quasi-static limit, i.e., when the ratio $\Lambda / \lambda \ll 1$, a simple homogenization treatment leads to the two following effective indices for the TE and TM states of polarization (see Born and $\mathrm{Wolf}^{9}$ ):

$$
\begin{aligned}
n_{\mathrm{eff}, 0}^{\mathrm{TE}} & =\left[F n_{a}{ }^{2}+(1-F) n_{b}{ }^{2}\right]^{1 / 2}, \\
n_{\mathrm{eff}, 0}^{\mathrm{TM}} & =\left[\frac{n_{a}{ }^{2} n_{b}{ }^{2}}{F n_{b}^{2}+(1-F) n_{a}^{2}}\right]^{1 / 2},
\end{aligned}
$$

where $n_{a}$ and $n_{b}$ are the structure's real indices, and where $F$ is the filling factor (ratio of the width of grating ridges to the period, $a / \Lambda$ ) (see Fig. 2). This straightforward approach is called the zero-order effective-medium theory (EMT0). When the ratio $\Lambda / \lambda$ is no longer negligible, the latter closed-form expressions for the effective refractive indices are no longer correct. In such cases, the second-order effective- 

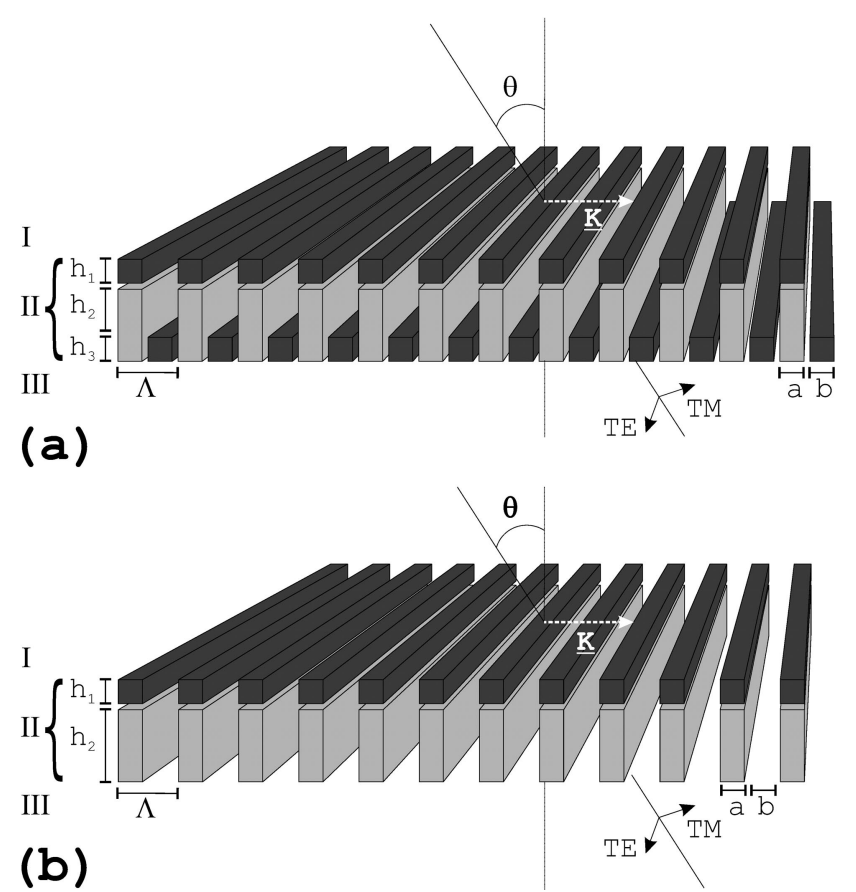

Fig. 2. ZOG schematic presenting the main parameters of the grating: the grating vector $|\underline{K}|=2 \pi / \Lambda$ perpendicular to the grating lines with $\Lambda$ being the period, the grating depth $h$, and the filling factor $F=a / \Lambda$. (a) Two AR-layer design, where the AR-layer thickness is $h_{1}=h_{3}$. and $h_{2}+h_{3}=h$. (b) One AR-layer design, where the AR-layer thickness is $h_{1}$ and $h_{2}=h$. The grating (medium II) is surrounded by the media I (superstrate) and III (substrate). The incident light, making an angle $\theta$ with the grating normal, can be decomposed in its TE (transverse electric) and TM (transverse magnetic) states of polarization.

medium theory (EMT2), which is deduced from the electromagnetic propagation in stratified media theory, allows us to derive these expressions for the effective indices ${ }^{10}$ :

$$
\begin{aligned}
n_{\mathrm{eff}, 2}{ }^{\mathrm{TE}}= & {\left[\left(n_{\mathrm{eff}, 0}{ }^{\mathrm{TE}}\right)^{2}+\frac{1}{3}\left(\frac{\Lambda}{\lambda}\right)^{2} \pi^{2} F^{2}(1-F)^{2}\right.} \\
& \left.\times\left(n_{a}{ }^{2}-n_{b}{ }^{2}\right)^{2}\right]^{1 / 2}, \\
n_{\mathrm{eff}, 2}{ }^{\mathrm{TM}}= & {\left[\left(n_{\mathrm{eff}, 0}{ }^{\mathrm{TM}}\right)^{2}+\frac{1}{3}\left(\frac{\Lambda}{\lambda}\right)^{2} \pi^{2} F^{2}(1-F)^{2}\right.} \\
& \left.\times\left(\frac{1}{n_{a}{ }^{2}}-\frac{1}{n_{b}{ }^{2}}\right)^{2}\left(n_{\mathrm{eff}, 0}{ }^{\mathrm{TM}}\right)^{6}\left(n_{\mathrm{eff}, 0}^{\mathrm{TE}}\right)^{2}\right]^{1 / 2} .
\end{aligned}
$$

In addition to the dependence on the wavelength, we also note the dependence of the effective indices versus other parameters available in a design procedure: the grating period $\Lambda$, the filling factor $F$, and the grating real indices $n_{a}$ and $n_{b}$ (see Fig. 2). The wavelength dependence of the effective indices is consequently also found in the form birefringence $\Delta n_{\text {form }}$. This phenomenon is called the dispersion of form birefringence. We must emphasize the term "form." Indeed, this property appearing just before the fron-

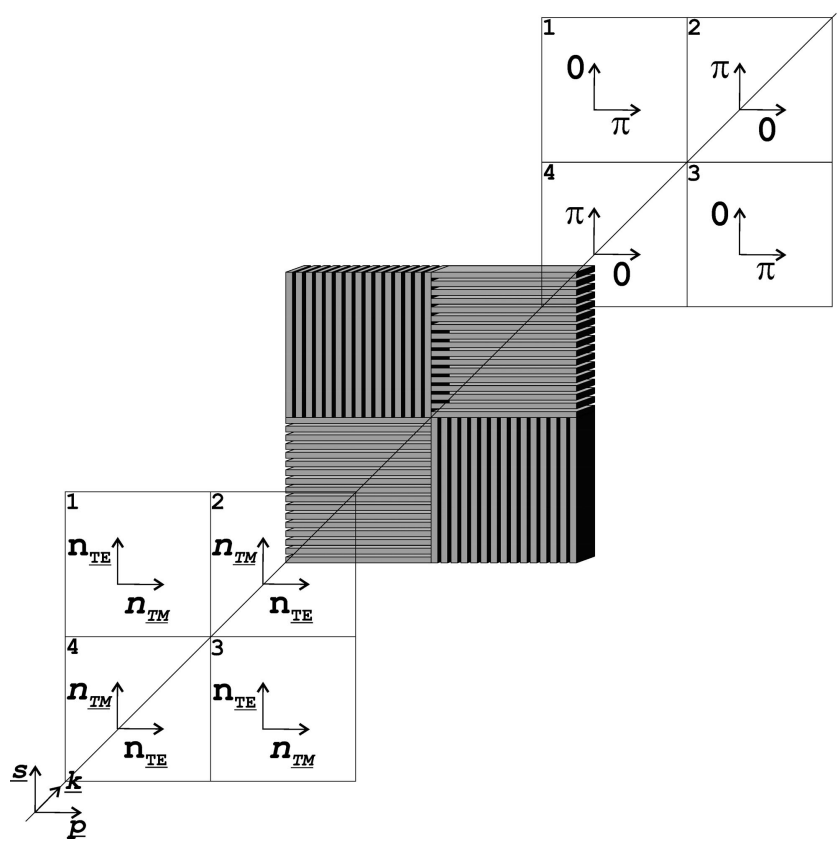

Fig. 3. 4QZOG implementation: $\underline{s}$ and $\underline{p}$ are the vectorial complex amplitude components of the incoming light of wave vector $\underline{k}$. In each of the four quadrants, the $\underline{s}$ and $\underline{p}$ global polarization states are decomposed in the corresponding $\mathrm{TE}_{i}$ and $\mathrm{TM}_{i}$ vectorial complex amplitudes according to the local grating line orientations $(i$ is the quadrant number). Two effective indices $n_{\mathrm{TE}_{i}}$ and $n_{\mathrm{TM}_{i}}$ can be assigned to the corresponding perpendicular polarization states. The four gratings engraved on a unique substrate are strictly identical and implemented in the following way: two of them in two quadrants along one diagonal are rotated by $90^{\circ}$ around their normals with respect to the two others. This antisymmetrical configuration achieves the FQPM particular focal plane $\pi$-phase distribution (see text for explanations).

tier of the resonant domain is essentially given by the geometry, no longer only by the intrinsic characteristics of the materials. The key point is that, by carefully controlling the geometry of the grating structure, we will be able to tune the form birefringence to compensate for the hyperbolic dependence of the phase shift $[\propto 1 / \lambda$, see Eq. (4)] and thus make it achromatic. This is a good example of refractiveindex engineering.

\section{FQPM Implementation: the Four-Quadrant ZOG (4QZOG)}

The implementation of the ZOG phase shifters into the FQPM is straightforward and represented in Fig. 3. Let $\underline{s}$ and $\underline{p}$ be the vectorial complex amplitude components of the incoming light. In each of the four quadrants, the $\underline{s}$ and $\underline{p}$ global polarization states can be decomposed in the $\mathrm{TE}_{i}$ and $T M_{i}$ vectorial complex amplitudes according to the line orientations of the local grating in the $i$ th quadrant, with $i=1, \ldots, 4$. Indeed, the convention under normal incidence specifies that the electric field of the TE and TM components vibrates parallel and perpendicular to the grating lines, respectively. We have shown above that two effective indices, $n_{\mathrm{TE}_{i}}$ and $n_{\mathrm{TM}_{i}}$, can be assigned to the corresponding perpendicular polarization states. 
Let us assume now that (i) the four gratings engraved on a unique substrate are strictly identical and (ii) they are implemented in the following way: two of them in two quadrants along one diagonal are rotated by $90^{\circ}$ around their normal with respect to the two others.

The consequence of the first hypothesis is that the effective indices $n_{\mathrm{TE}_{i}}$ and $n_{\mathrm{TM}_{i}}$ are identical in each quadrant, i.e., $n_{\mathrm{TE}_{i}}=n_{\mathrm{TE}}$ and $n_{\mathrm{TM}_{i}}=n_{\mathrm{TM}}$, with $i=1$, $\ldots, 4$. The second one gives this particular antisymmetrical configuration (Fig. 3) with the following result. If, by construction,

$$
\begin{aligned}
\Delta \phi_{\mathrm{TE}_{i}-\mathrm{TM}_{i}} & =\frac{2 \pi}{\lambda} h \Delta n_{\mathrm{TE}_{i}-\mathrm{TM}_{i}} \\
& =\frac{2 \pi}{\lambda} h \Delta n_{\mathrm{TE}-\mathrm{TM}} \approx \pi,
\end{aligned}
$$

then the potentially interfering parallel polarization states along the $\underline{s}$ component direction, for example, are phase shifted in the following way ( $j$ being an adjacent quadrant of $i$ ):

$$
\begin{aligned}
\Delta \phi_{\mathrm{TE}_{i}-\mathrm{TM}_{j}} & =\frac{2 \pi}{\lambda} h\left(n_{\mathrm{TE}_{i}}-n_{\mathrm{TM}_{j}}\right) \\
& =\frac{2 \pi}{\lambda} h\left(n_{\mathrm{TE}_{i}}-n_{\mathrm{TM}_{i}}\right) \\
& =\Delta \phi_{\mathrm{TE}_{i}-\mathrm{TM}_{i}} \approx \pi .
\end{aligned}
$$

The same goes for the $p$ component direction. Consequently, for the $s$ and $\underline{p}$ component directions, respectively, the FQPM particular focal plane $\pi$-phase distribution is achieved, i.e., adjacent quadrants are in phase opposition. Therefore, it works in natural light. We will call this new structure the fourquadrant ZOG (4QZOG). It must be noted that the precision on the perpendicularity between the gratings is directly related to the nulling performance. It has to be finely controlled.

\section{Design of the $4 Q Z O G$}

Effective-medium theories are qualitatively interesting to understand the underlying physical phenomena, but only deal with zero-order diffracted waves and disregard the evanescent higher-order ones. To obtain the exact response of this kind of structure, they have to be taken into account together with external media influences in terms of multiple-beam interferences, just as in the so-called rigorous coupled wave analyses (RCWA). ${ }^{11}$ Moreover, this theory gives, in addition to the phase, the intensity of the diffracted waves (diffraction efficiencies).

\section{A. Preliminary Considerations}

The design procedure consists of the optimization of the $\pi$ phase-shift quality within a given spectral range for a well-chosen material. It also requires the equalization of the interfering fluxes, which is difficult to achieve. Indeed, the difference between the effective indices that induces the form birefringence is also responsible for the resultant unequal TE/TMFresnel reflection coefficients at the interfaces between the modulated region and the external media. The merit function to be minimized is the null depth $N(\lambda)$. The latter is directly related to the phase-shift error with respect to $\pi, \varepsilon(\lambda)=\Delta \phi_{\mathrm{TE}-\mathrm{TM}}(\lambda)-\pi$, and the flux ratio $q(\lambda)=n_{\mathrm{TE}}(\lambda) / n_{\mathrm{TM}}(\lambda)$ :

$$
N(\lambda)=\frac{[1-\sqrt{q(\lambda)}]^{2}+\varepsilon(\lambda)^{2} \sqrt{q(\lambda)}}{[1+\sqrt{q(\lambda)}]^{2}} .
$$

We have investigated several ways to ensure a proper flux balance, i.e., $q \approx 1$. The principle is always the same and consists of trying to minimize the index discontinuities (impedance matching) at the interfaces by the following:

(i) Varying the grating profile: The structure can have trapezoidal profiles instead of rectangular ones. It reduces the parasitic reflection but keeps the anisotropy and a sufficient form birefringence dispersion up to a certain point.

(ii) "Sandwiching" the grating: The superstrate material can be chosen to avoid the brutal air-ZOG interface. ${ }^{12}$ It is conceptually interesting and gives good numerical results, but presents extreme manufacturing challenges, such as how to keep the grating openings clear.

(iii) Depositing a classical antireflective (AR) layer: The ZOG is AR treated with a single $\lambda / 4$ layer. The latter settles in the bottom of the openings and on the top of the grating ridges [Fig. 2(a)]. This leads to an original configuration, a three-layer grating with a complex behavior governed by diffraction. We demonstrate that this particular structure can be optimized to give interesting results.

Currently, subwavelength gratings are manufactured more readily for the near-infrared and midinfrared domains because there are still difficulties in imprinting submicrometer features. Hopefully, the near-term potential applications (VLT-PF, for example) of this technology concern this wavelength domain. We will focus on the astrophysical band filters $H(1.475-1.825 \mu \mathrm{m}), K(2-2.4 \mu \mathrm{m})$, and $N(9.3-11.6 \mu \mathrm{m})$. We have to choose among the restricted list of infrared materials, but the ZOG technology is sufficiently flexible to accommodate the majority of them. We will present results for diamond, zinc selenide (ZnSe), cadmium telluride $(\mathrm{CdTe})$, silicon $(\mathrm{Si})$, and germanium $(\mathrm{Ge})$. This choice is justified because these selected materials are common in infrared applications, they cover a large refractive-index spectrum (2-4), and their etching processes are well known.

\section{B. 4QZOG Theoretical Analysis}

The indices for diamond, ${ }^{13} \mathrm{ZnSe},{ }^{14} \mathrm{CdTe},{ }^{15} \mathrm{Si},{ }^{15}$ and Ge (Ref. 15) will be taken from the following repre- 
Table 1. Coefficients for Material Refractive-Index Representations

\begin{tabular}{cccccc}
\hline Coefficient & Diamond & ZnSe & CdTe & Si & Ge \\
\hline$A$ & 1 & 1 & $-2.373 \times 10^{-4} T+3.8466$ & $1.600 \times 10^{-4} T+3.431$ & $-6.040 \times 10^{-3} T+11.05128$ \\
$B$ & 0.3306 & 4.46395 & $8.057 \times 10^{-4} T+3.2215$ & $-2.643 \times 10^{-2}$ & $9.295 \times 10^{-3} T+4.00536$ \\
$C$ & 30625 & 0.0404 & $-1.10 \times 10^{-4} T+0.1866$ & $4.324 \times 10^{-3}$ & $-5.392 \times 10^{-4} T+0.599034$ \\
$D$ & 4.3356 & 0.46132 & $-2.160 \times 10^{-2} T+12.718$ & $-3.194 \times 10^{-4}$ & $4.151 \times 10^{-4} T+0.09145$ \\
$E$ & 11236 & 0.1538 & $-3.160 \times 10^{1} T+18753$ & $8.835 \times 10^{-6}$ & $1.51408 T+3426.5$ \\
$F$ & - & 2.88289 & - & - & - \\
$G$ & - & 2213.5 & - & - & - \\
\hline
\end{tabular}

sentations:

$$
\begin{aligned}
n_{\text {diamond,ZnSe,CdTe,Ge }}(\lambda)= & \left(A+\frac{B \lambda^{2}}{\lambda^{2}-C}+\frac{D \lambda^{2}}{\lambda^{2}-E}\right. \\
& \left.+\frac{F \lambda^{2}}{\lambda^{2}-G}\right)^{1 / 2}, \\
n_{\mathrm{Si}}(\lambda)= & A+B \lambda+C \lambda^{2}+D \lambda^{3}+E \lambda^{4} .
\end{aligned}
$$

The corresponding coefficients are given in Table 1. We assume an ambient temperature $(T=298 \mathrm{~K})$, keeping in mind that corrections are needed if $T$ is different. Note that $\lambda$ is expressed in micrometers in all representations except for diamond, where it is expressed in nanometers.

We have performed simulations for the $H, K$, and $N$ bands, assuming in each case a ZOG AR treated with a $\mathrm{YF}_{3}$ layer. ${ }^{16}$ Absorption and dispersion of $\mathrm{YF}_{3}$ have been taken into account (see Fig. 13 in Lemarquis et al. ${ }^{17}$ ). We chose $\mathrm{YF}_{3}$ as a coating material, but several other possibilities exist such as $\mathrm{BaF}_{2}$ and $\mathrm{LaF}_{2}$, which could be better choices according to the substrate index. The grating structure in all these simulations consists of a single rectangular profile discretized into three levels to take the deposited AR layer into account [Fig. 2(a)]. The results are always discussed in terms of the null depth [Eq. (9)]. Despite the flexibility in the design of the $4 \mathrm{QZOG}$, there will be a compromise on the substrate refractive properties. Indeed, the dispersion of the form birefringence is proportional to the raw index $n$. Practically, $n$ must be greater than approximately 2 to sufficiently compensate for the wavelength hyperbolic dependence of the phase shift [Eq. (4)] and thus achromatize it. Unfortunately, the intensity of the parasitic reflection phenomenon will also increase accordingly. We have already discussed the flux imbalance problem, but the Fresnel reflection will also be responsible for a so-called second-order ghost in the final coronagraphic image. This ghost comes from the double reflection on the back face of the substrate and on the internal interface of the AR-treated ZOG. Its intensity can be quantified assuming a reasonable AR performance for the back side of the component, i.e., about $0.5 \%$ of reflectivity.

We can see in Fig. 4 that the ghost intensity level significantly increases with the substrate refractive index. We also note that the rough null depth performance is inversely proportional to the material index. Indeed, our AR-layer solution for the differential flux problem shows here its limitations. For this reason, and for the sake of simplicity, we will restrain our analysis to ZnSe and diamond, both possessing the lowest-index values (near 2.4).

Results for both selected materials in terms of null depth are excellent either for the $H, K$, or $N$ bands (Figs. 5, 6, and 13). Indeed, the mean null depth performances are at the $10^{-5}$ level over the whole spectral range of the three considered band filters. Without losing generality, we shall restrict further analysis to the particular case of the $K$ band. We have explicated the performances in terms of phase-shift quality and differential transmittances between the TE and the TM states of polarization (Figs. 7 and 8). The standard deviation of the phase around $\pi$ is only $7 \times 10^{-3} \mathrm{rad} \mathrm{rms}$, which is good and indicates a small residual chromaticity. As far as the transmittances through the ZOG are concerned (zero-order diffrac-

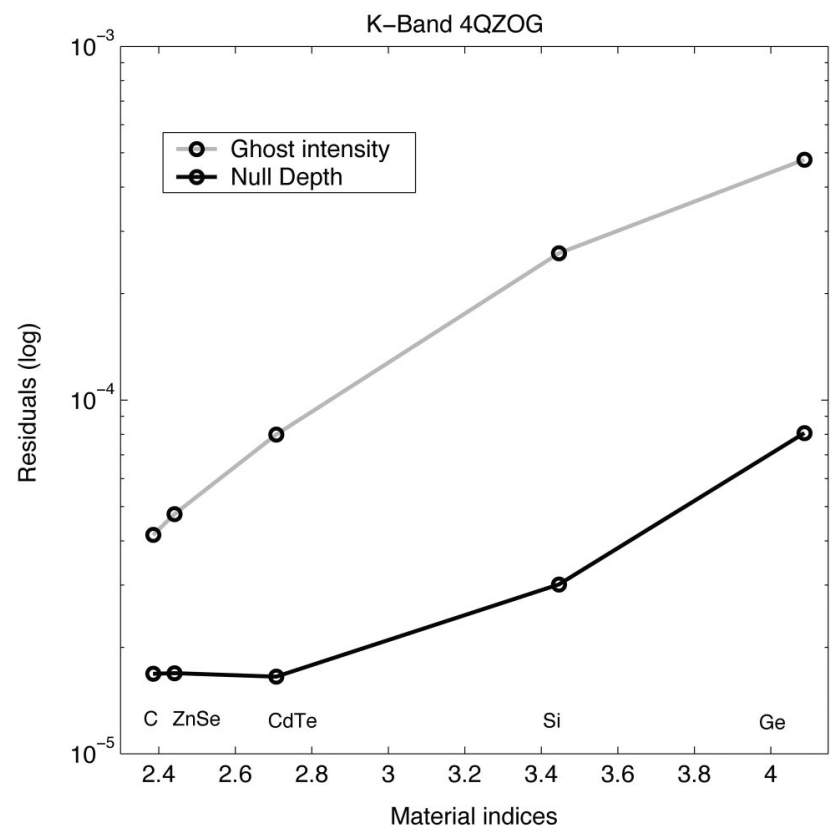

Fig. 4. Design level of performance according to the substrate refractive index $n$. The ghost intensity level increases significantly with $n$. We also note that the rough null depth performance is inversely proportional to $n$. The best compromise is therefore for the low-index values. 


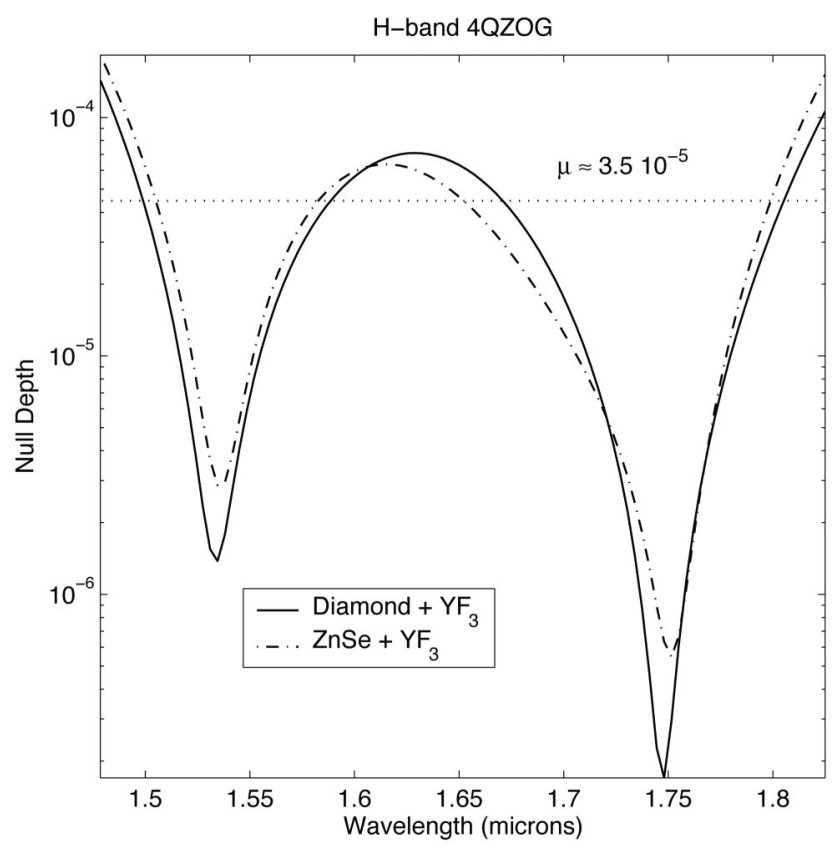

Fig. 5. $H$-band 4QZOG null depth (logarithmic scale) vs wavelength. The continuous curve is for the diamond $\mathrm{YF}_{3}$ AR-coated 4QZOG, the dashed curve for the $\mathrm{ZnSe} \mathrm{YF}_{3} \mathrm{AR}$-coated one. $\mu \approx 3.5$ $\times 10^{-5}$ is the mean null depth over the whole $H$ band.

tion efficiencies in transmission for the TE and TM components), we note an important absorption due to the $\mathrm{YF}_{3}$ layer. This energy leakage affects the optical throughput of the system but favorably reduces the ghost level by absorbing a fraction of the internal higher-order parasitic reflections.

The grating parameters of the $K$-band optimized

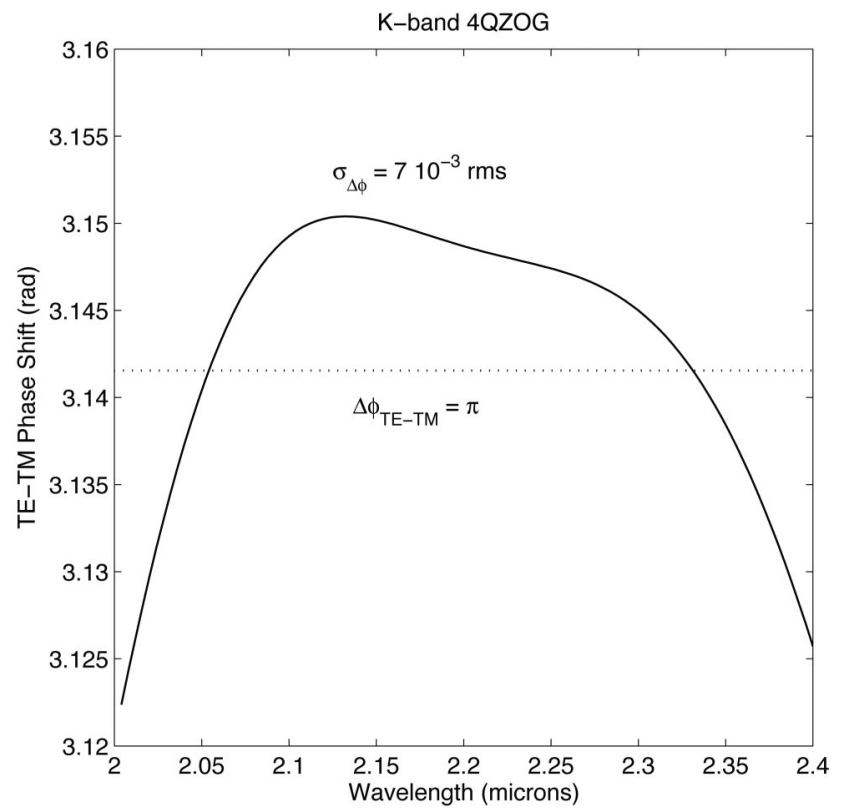

Fig. 7. $K$-band $4 \mathrm{QZOG}$ phase shift between the TE and TM polarization states vs wavelength: the phase-shift quality is quantified by the phase-shift standard deviation $\sigma_{\Delta \phi}$ (the less, the better), which is at the very good level of $7 \times 10^{-3} \mathrm{rad} \mathrm{rms}$.

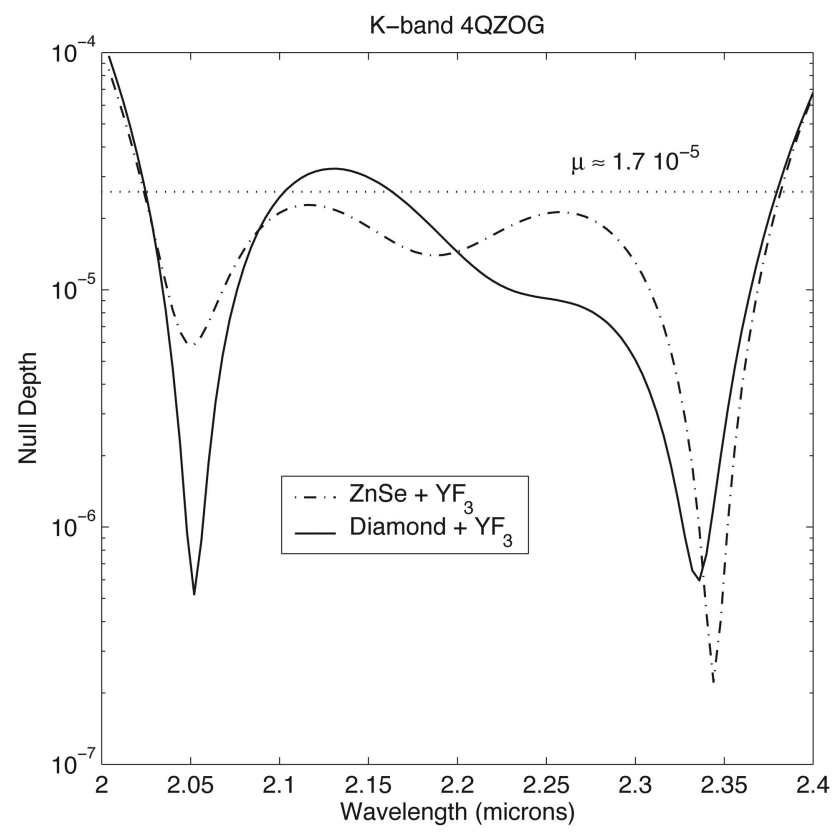

Fig. 6. $K$-band 4QZOG null depth (logarithmic scale) vs wavelength. The continuous curve is for the diamond $\mathrm{YF}_{3}$ AR-coated 4QZOG, the dashed curve for the $\mathrm{ZnSe} \mathrm{YF}_{3}$ AR-coated one. $\mu \approx 1.7$ $\times 10^{-5}$ is the mean null depth over the whole $K$ band.

solutions for the ZnSe and diamond materials are given in Table 2. All the fabrication aspects will be considered in Section 5. These optimal parameter sets have been calculated by implementing the simplex search method $^{17}$ with our RCWA algorithm. One

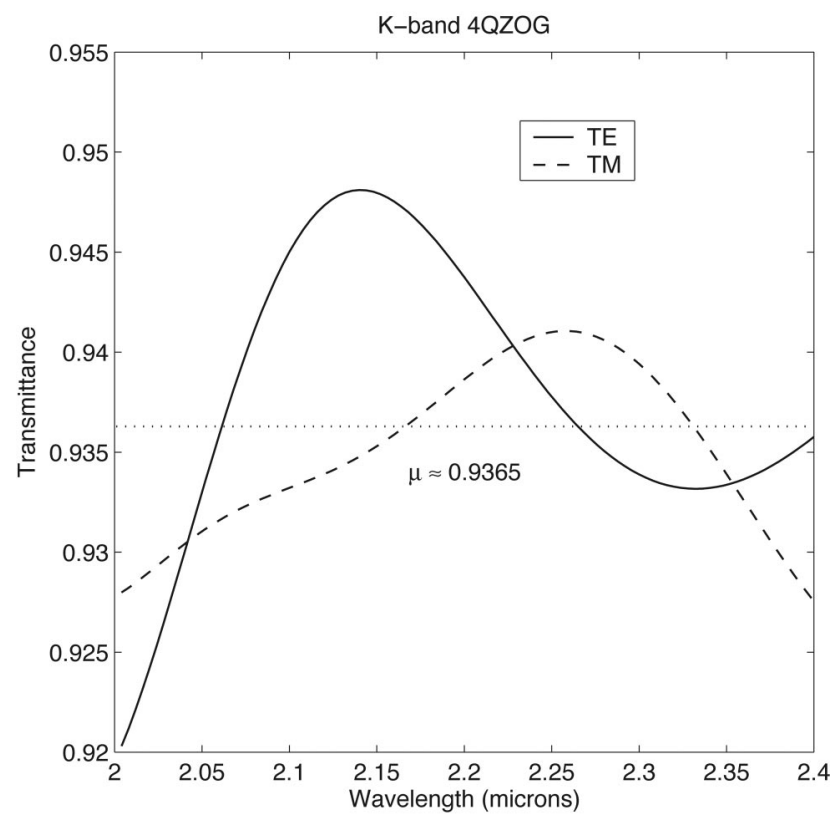

Fig. 8. $K$-band 4QZOG transmittances (zero-order diffraction efficiencies in transmission for the TE and TM components) vs wavelength, including the mean optical throughput of the component, which is $\approx 93.6 \%$, taking absorption into account. The amplitude peak-to-valley variations for the two polarizations TE and TM over the whole band range from $1.5 \%$ to $3 \%$. 
Table 2. K-Band Diamond and ZnSe 4QZOG Parameters (Two AR-Layer Design)

\begin{tabular}{ccc}
\hline \multicolumn{1}{c}{ Parameters } & Diamond & ZnSe \\
\hline Grating period $\Lambda$ & $0.740 \mu \mathrm{m}$ & $0.708 \mu \mathrm{m}$ \\
Grating depth & $3.238 \mu \mathrm{m}$ & $3.116 \mu \mathrm{m}$ \\
$h\left(=h_{2}+h_{3}\right)$ & & \\
Grating filling factor $F$ & $70 \%$ & $72 \%$ \\
$\quad(=a / \Lambda)$ & & $404 \mathrm{~nm}$ \\
$\mathrm{YF}_{3}$ AR-layer thickness & $423 \mathrm{~nm}$ & \\
$h_{\mathrm{AR}}\left(=h_{1}=h_{3}\right)$ & & \\
\hline
\end{tabular}

can see that only minor corrections are needed between the $\mathrm{ZnSe}$ and the diamond designs; though their dispersions are quite different, their indices are very close. Parameters for other bands may be derived by simple scaling operations, with little corrections to take the index dispersion and/or absorption of the substrate and AR layer again into account.

In conclusion, the results we obtained, taking phase defects and amplitude inequalities into account, are very good with deep $\left(\approx 10^{-5}\right)$ average nulls over the considered wavelength bands. This level of performance is sufficient in the case of phase coronagraphy, as we will see in Section 6 .

\section{Tolerancing and Manufacturing}

The filling factor $(F)$ is the determinant parameter of the grating structure and the most difficult to control during the fabrication process. Its influence can be analyzed by varying it and finding the best solutions in terms of the free parameters that remain; the grating period, depth, and the AR-layer thickness. Figure 9 shows the optimal configuration null depth as a function of the grating filling factor $F$. This calculation clearly points out that the best solutions occur for the largest $F$ with some local minima. The corresponding parameter adjustments are given in Fig. 10, where, for each $F$, the optimal period and depth have been recomputed. We logically note that the updated depth increases with $F$. Indeed, the form birefringence that can be considered as an optical anisotropy is inversely proportional to $F$, whereas its wavelength dispersion increases accordingly. Consequently, a given phase shift will be obtained for a deeper grating. These considerations illustrate the design's strong sensitivity to the parameters.

To assess the actual feasibility of the component fabrication, we have performed several tolerance simulations. By varying each parameter independently around an optimal solution, the specifications would impose an unrealistic tight control of the profile and depth of the grating (at the nanometer level). We have adopted a more physical approach that requires the scanning of the parameter space searching affordable solutions in terms of a well-chosen merit figure. In our application, the latter is the null depth as defined in Eq. (9) and integrated over the whole considered wavelength band. These time-consuming RCWA calculations have led to interesting results (Fig. 11), demonstrating that the manufacturing is

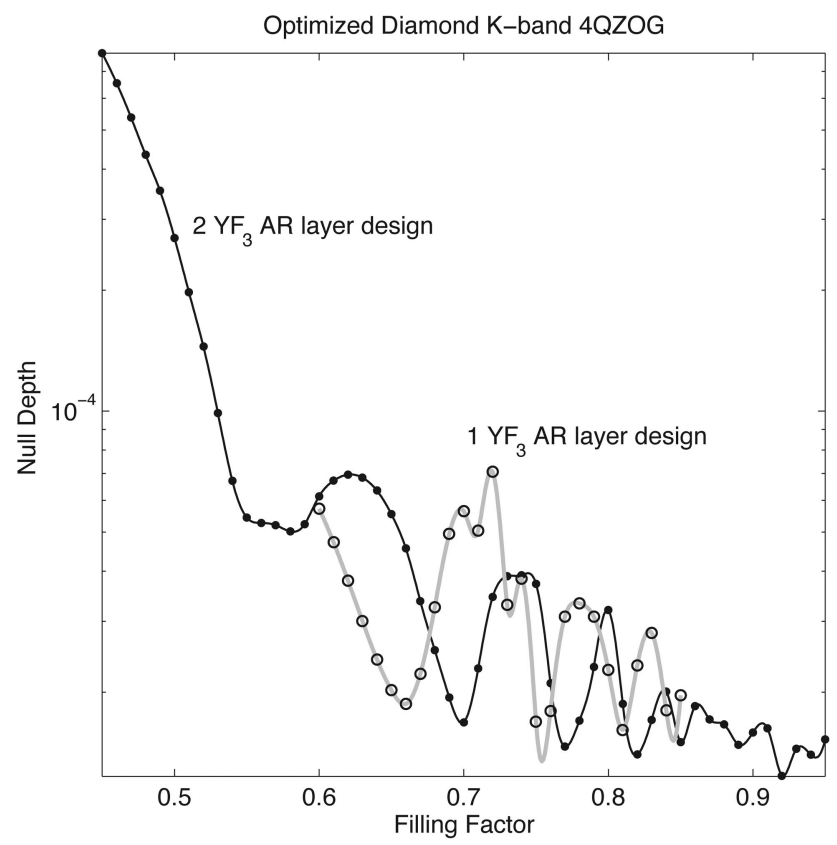

Fig. 9. Null depth (logarithmic scale) versus the grating filling factor $(F)$. An optimal solution has been calculated at each $F$ value. We note that best null depths occur for the largest $F$ values with some local minima. We have overplotted in gray the one AR-layer case in which we notice a tiny average degradation of the null depth (negligible in our application) but a nonnegligible shift in optimal filling factors for the manufacturing.

within the reach of current microelectronic technology. The fabrication implies the traditional lithography step defining the profile mask either with direct laser writing or $e$-beam lithography and then the transfer of the pattern into the substrate by plasma

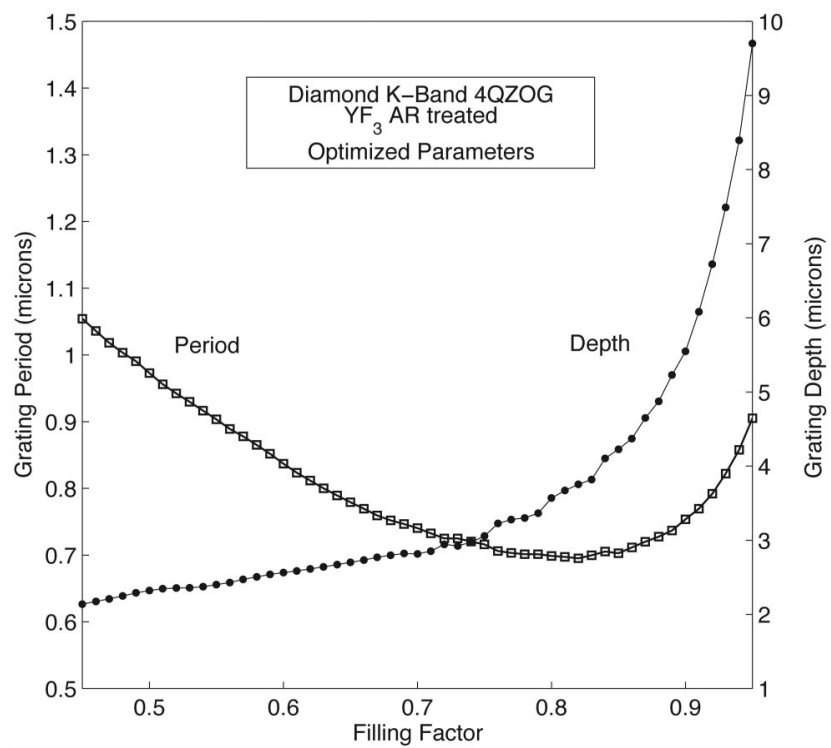

Fig. 10. Double plot of the grating depth and period vs the filling factor $(F)$. The calculations show the optimal recomputed grating period (left) and depth (right) for the best null depth at each $F$. We note that the depth increases with $F$. The period shows a parabolic behavior. 


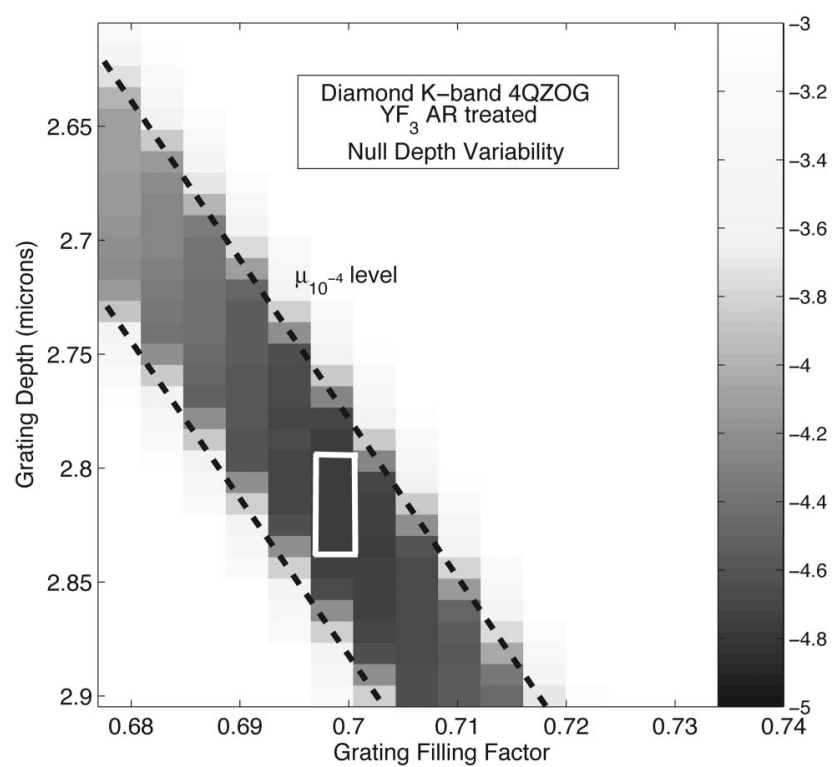

Fig. 11. 4QZOG null depth sensitivity (logarithmic scale) to the grating depth $h$ and filling factor $F$ parameter variations. Each point in this figure represents the average null depth over the whole $K$ band for a given set of $h$ and $F$ but retaining the best solution among several AR-layer thicknesses (with a $2 \%$ tolerance) to accommodate this a posteriori possibility of correction.

etching. These techniques are more adapted to small filling factors. This is the reason why we have always privileged the lowest ones in our optimal results, i.e., around $F \approx 70 \%$ (see Fig. 11). It is to be noted that such structures with similar aspect ratios (the aspect ratio is the ratio between the grating depth and the width of the grating ridges) have already been successfully produced in silicon ${ }^{18}$ using these methods.

Assuming a classical realistic resolution of $10 \mathrm{~nm}$ in thickness (2\%) for an a posteriori correction with the AR layer, we can ensure a grating etching depth tolerance of about $100 \mathrm{~nm}$ at the null depth level of $10^{-4}$ (affordable solution). This result also assumes a perfect a priori metrology of the photolithographic mask using either the scanning electron microscope or the atomic force microscope. These conclusions have led us to seriously consider the fabrication of the component that is under study with the collaboration of "Adamantis AB" at the Angström Laboratory (University of Uppsala, Sweden). Indeed, they have developed a very robust and well-proven process for diamond microstructuring. ${ }^{19,20}$ The preliminary manufacturing assessments have already led us to consider the possibility of depositing the AR layer on the bare substrate before etching. In such a case, there will only be one layer settling at the top of the grating ridges [see Fig. 2(b)]. Corresponding numerical simulations have only tackled a tiny average degradation of the final performances but negligible in our application (Fig. 9). The reason is that the greatest contribution to the parasitic Fresnel reflections comes from the first interface (air-ZOG) where the index discontinuities are the largest. It should be noted that in the one AR-layer design, there is a

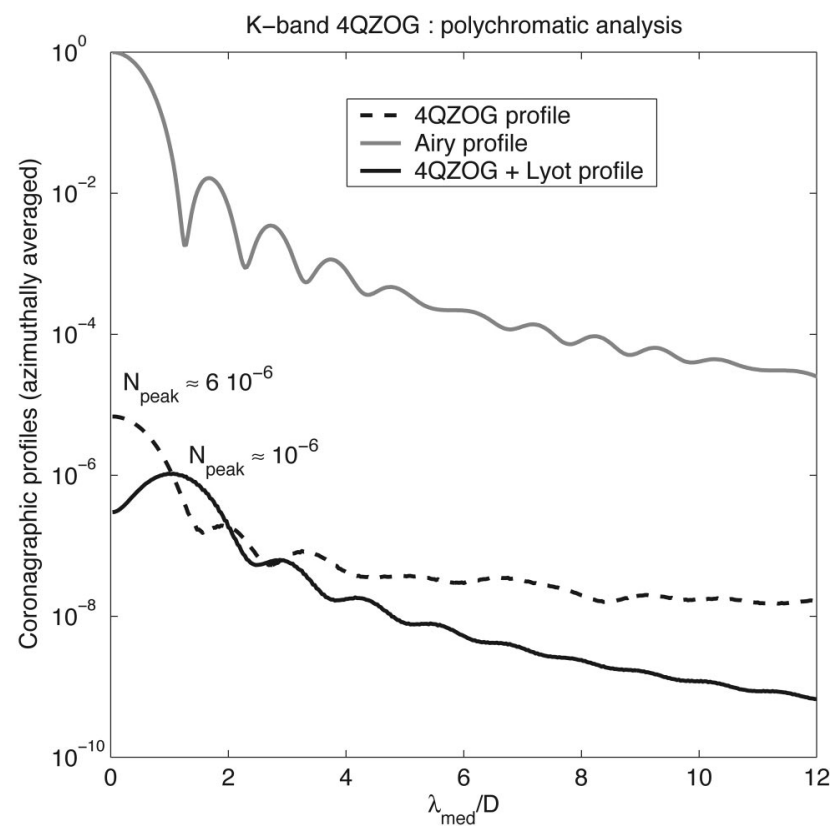

Fig. 12. $K$-band 4QZOG coronagraphic profile (logarithmic scale) vs the angular separation in $\lambda_{\text {med }} / \mathrm{D}$ unity, $\mathrm{D}$ being the telescope diameter. The gray curve corresponds to the polychromatic Airy disk. The dashed curve represents the wide band 4QZOG azimuthally averaged profile where the peak-to-peak null depth is about $6 \times 10^{-6}$. The continuous curve corresponds to the previous case with a tiny circular Lyot opaque mask $\left(0.55 \lambda_{\text {med }} / D\right.$ of diameter $)$ to get rid of the chromatic residuals and ghost image. The peak-topeak null depth is approximately $10^{-6}$.

nonnegligible correction of the other grating parameters with respect to the two-layer one. In conclusion, both designs work and the choice belongs to the manufacturer.

\section{Coronagraphic Simulations}

We have performed polychromatic coronagraphic simulations for the $K$-band 4QZOG. These calculations try to simulate the broadband response of the component on a perfect optical bench. It thus only tackles chromatic effects. We have linked the output results of our RCWA code in terms of phase and amplitude transmittances to a traditional Fourier propagation coronagraphic simulator. ${ }^{21}$ The results are excellent (Fig. 12) and are in good agreement with the corresponding null depth curve (Fig. 6).

The polychromatic null depth on the Airy peak is about $6 \times 10^{-6}$. The $10^{-7}$ speckle level is very quickly reached at $2 \lambda_{\text {med }} / D \quad\left(\lambda_{\text {med }}=2.2 \mu \mathrm{m}\right)$, where $D$ is the telescope diameter. Better performances $\left(10^{-6}\right.$ on the peak) can be achieved by placing at the center of the component, a tiny ${ }^{4}$ circular Lyot opaque mask of $0.55 \lambda_{\text {med }} / D$, which is the diameter encircling half of the Airy disk energy. By doing this, we remove a large part of the 4QZOG chromatic residuals and also prevent the annoying ghost problem to a certain point. These performances are comfortably 1 order of magnitude above the $H$-band/K-band VLT-PF specification. Indeed, the main limitation for ground-based observations comes from the atmospheric turbulence 


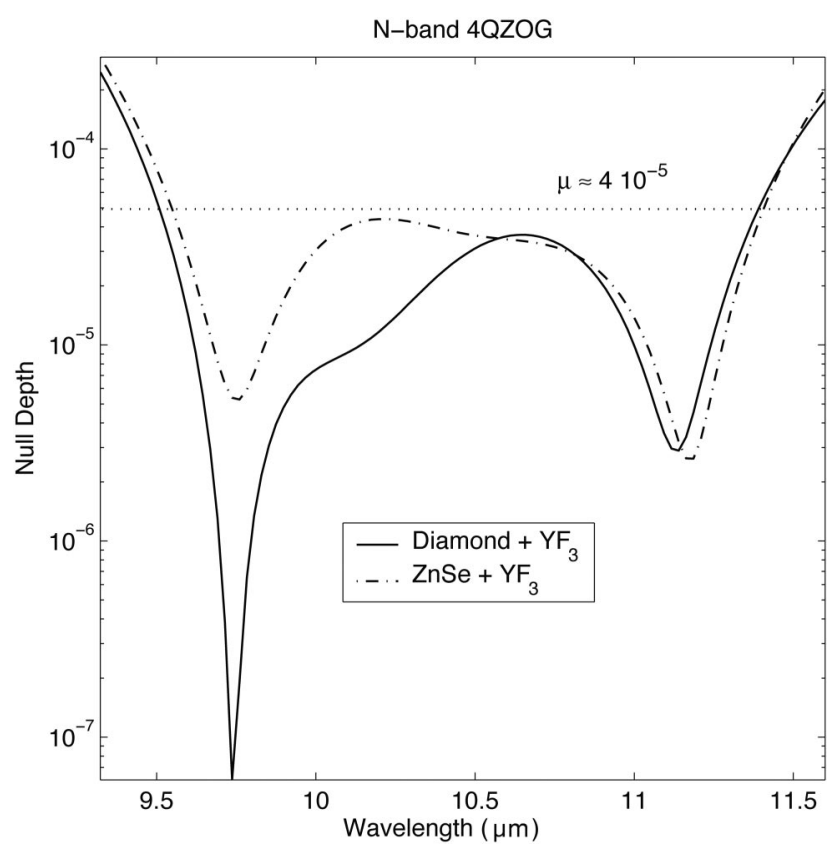

Fig. 13. $N$-band 4QZOG null depth (logarithmic scale) versus wavelength. The continuous curve is for the diamond $\mathrm{YF}_{3} \mathrm{AR}$ -

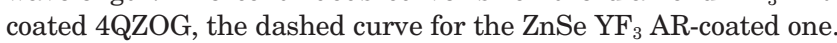
$\mu \approx 4 \times 10^{-5}$ is the mean null depth over the whole $K$ band.

residuals (the so-called speckles) after adaptive optics corrections of the incoming fluctuating wavefronts. For this independent reason, the baseline dynamic will be limited to a maximum null depth of $10^{-4}$.

Another potential application of the 4QZOG could be an infrared interferometer in the $N$ band for NASA's Terrestrial Planet Finder and ESA's InfraRed Space Interferometer-DARWIN missions; the main limitation of the nulling interferometer will be the leakages from the partially resolved stellar diameter. Therefore, the performance obtained in our theoretical simulations (Fig. 13) would again be sufficient.

\section{Conclusion}

We have presented an original solution to the chromaticity problem of the $\pi$ phase shift for the FQPM coronagraph by using subwavelength gratings (ZOGs). Indeed, the birefringence of ZOGs can be controlled by the geometry to compensate for the hyperbolic dependence of the phase shift and intrinsic material dispersions. We call this new design the "4QZOG." The results (null depth $\approx 10^{-5}$ ) are within ground-based instrument specifications for exoplanet detection (VLT-PF) and could also be envisaged for future ambitious space-based missions (TPF/ DARWIN). We have also demonstrated the feasibility of the component fabrication with realistic tolerances. Its manufacturing is under assessment by "Adamantis AB" (Angström Laboratory, Uppsala University, Sweden). It will be the subject of a forthcoming paper.
D. Mawet acknowledges the financial support of the Belgian "Fonds pour la formation à la Recherche dans l'Industrie et dans l'Agriculture." P. Riaud and J. Surdej acknowledge the financial support of the "Pôle d'Attraction Inter-Universitaire."

\section{References}

1. B. Lyot, "A study of the solar corona and prominences without eclipses," Mon. Not. R. Astron. Soc. 99, 580-594 (1939).

2. B. A. Smith and R. Terrile, "A circumstellar disk around Beta Pictoris," Science 226, 1421-1424 (1984).

3. M. J. Kuchner and D. M. Spergel, "Notch-filter masks: practical image masks for planet-finding coronagraphs," Astrophys. J. 594, 617-626 (2003).

4. F. Roddier and Cl. Roddier, "Stellar coronagraph with phase mask," Publ. Astron. Soc. Pac. 109, 815-820 (1997).

5. D. Rouan, P. Riaud, A. Boccaletti, Y. Clénet, and A. Labeyrie, "The four-quadrant phase-mask coronagraph. I. Principle," Publ. Astron. Soc. Pac. 112, 1479-1486 (2000).

6. P. Riaud, J. Baudrand, A. Boccaletti, and D. Rouan, "The fourquadrant phase-mask coronagraph. III. Laboratory performance," Publ. Astron. Soc. Pac. 115, 712-719 (2003).

7. D. Gratadour, D. Rouan, A. Boccaletti, P. Riaud, and Y. Clénet, "Four quadrant phase mask K-band coronagraphy of NGC 1068 with NAOS-CONICA at VLT," Astron. Astrophys. 429, 433-437 (2005).

8. D. Mouillet, T. Fusco, A.-M. Lagrange, and J.-L. Beuzit, "Planet Finder on the VLT: context, goals, and critical specifications for adaptive optics," EAS Publ. Ser. 8, 193-200 (2003).

9. M. Born and E. Wolf, Principles of Optics (Cambridge University Press, 1999), Chap. 15, pp. 837-840.

10. S. M. Rytov, "Electromagnetic properties of a finely stratified medium," Sov. Phys. JETP 2, 466-475 (1956).

11. M. G. Moharam and T. K. Gaylord, "Rigourous coupled-wave analysis of planar grating diffraction,” J. Opt. Soc. Am. 71, 811-818 (1981).

12. H. Kikuta, Y. Ohira, and K. Iwata, "Achromatic quarter-wave plates using the dispersion of form birefringence," Appl. Opt. 36, 1566-1572 (1997).

13. F. P. Bundy, "Melting point of graphite at high pressure: heat of fusion," Science 137, 1055-1057 (1962).

14. W. J. Tropf, "Temperature-dependent refractive index models, for $\mathrm{BaF}_{2}, \mathrm{CaF}_{2}, \mathrm{MgF}_{2}, \mathrm{SrF}_{2}, \mathrm{LiF}, \mathrm{NaF}, \mathrm{KCl}, \mathrm{ZnS}$, and $\mathrm{ZnSe}$, Opt. Eng. 34, 1369-1373 (1995).

15. G. J. Hawkins, "Spectral Characterisation of Infrared Optical Materials and Filters," Ph.D. dissertation (University of Reading, United Kingdom, 1998).

16. F. Lemarquis, G. Marchand, and C. Amra, "Design and manufacture of low-absorption $\mathrm{ZnS}_{\mathrm{n}} \mathrm{YF}_{3}$ antireflection coatings in the 3.5-16 $\mu \mathrm{m}$ spectral range," Appl. Opt. 37, 4239-4244 (1998).

17. J. C. Lagarias, J. A. Reeds, M. H. Wright, and P. E. Wright, "Convergence properties of the Nelder-Mead simplex method in low dimensions," SIAM J Optim 9, 112-147 (1998).

18. G. P. Nordin and P. C. Deguzman, "Broadband form birefringent quarter-wave plate for the mid-infrared wavelength region," Opt. Express 5, 163-168 (1999).

19. M. Karlsson and Fr. Nikolajeff, "Diamond micro-optics: microlenses and antireflection structured surfaces for the infrared spectral region," Opt. Express 11, 502-507 (2003).

20. M. Karlsson, K. Hjort, and Fr. Nikolajeff, "Transfer of continuous-relief diffractive structures into diamond by use of inductively coupled plasma dry etching," Opt. Lett. 26, 17521754 (2001).

21. P. Riaud, A. Boccaletti, D Rouan, F. Lemarquis, and A. Labeyrie, "The four-quadrant phase-mask coronagraph. II. Simulations," Publ. Astron. Soc. Pac. 113, 1145-1154 (2001). 